\title{
The effects of massage on delayed onset muscle soreness
}

\author{
J E Hilbert, G A Sforzo, T Swensen
}

Br J Sports Med 2003;37:72-75

See end of article for authors' affiliations

Correspondence to:

Thomas Swensen, PhD

Department of Exercise \&

Sport Sciences, Center for

Health Sciences, Ithaca,

NY 14850, USA;

tswensen@ithaca.edu

Accepted 7 May 2002
Objectives: The purpose of this study was to investigate the physiological and psychological effects of massage on delayed onset muscle soreness (DOMS).

Methods: Eighteen volunteers were randomly assigned to either a massage or control group. DOMS was induced with six sets of eight maximal eccentric contractions of the right hamstring, which were followed $2 \mathrm{~h}$ later by $20 \mathrm{~min}$ of massage or sham massage (control). Peak torque and mood were assessed at 2, 6, 24, and 48 h postexercise. Range of motion (ROM) and intensity and unpleasantness of soreness were assessed at 6,24, and $48 \mathrm{~h}$ postexercise. Neutrophil count was assessed at 6 and $24 \mathrm{~h}$ postexercise.

Results: A two factor ANOVA (treatment $v$ time) with repeated measures on the second factor showed no significant treatment differences for peak torque, ROM, neutrophils, unpleasantness of soreness, and mood ( $p>0.05$ ). The intensity of soreness, however, was significantly lower in the massage group relative to the control group at $48 \mathrm{~h}$ postexercise $(\mathrm{p}<0.05)$.

Conclusions: Massage administered $2 \mathrm{~h}$ after exercise induced muscle injury did not improve hamstring function but did reduce the intensity of soreness $48 \mathrm{~h}$ after muscle insult.
$\mathrm{D}$ elayed onset muscle soreness (DOMS) refers to the skeletal muscle pain that follows novel eccentric exercise. ${ }^{1-4}$ The intensity of soreness increases during the first $24 \mathrm{~h}$, peaks at 24-48 h, and subsides within 5-7 d postexercise. ${ }^{13}$ The sore muscles are described as feeling stiff, tender, and aching especially after palpitation or movement but these common symptoms rarely require medical attention. ${ }^{135}$ Researchers hypothesise that DOMS is related to muscle structural damage that is followed by ion imbalance, inflammation, and pain. ${ }^{1-35}$ Muscle damage includes disrupted sarcolemma, T-tubules, myofibrils, cytoskeletal protein, and sarcoplasmic reticulum (SR). ${ }^{15-7}$ Damage to the SR is particularly problematic, as it causes an ion imbalance that activates calpain, an enzyme that further degrades muscle proteins. ${ }^{589}$ Within $8 \mathrm{~h}$ of the initial injury, chemoattractants released by the damaged muscle tissue attract neutrophils, which adhere to the endothelium of nearby blood vessels in a process called adhesion or margination. ${ }^{2}{ }^{4}$ After adhesion, the neutrophils infiltrate the muscle tissue to phagocytosise damaged cells. If neutrophil function is not tightly controlled, healthy tissue is inadvertently destroyed and additional muscle damage occurs. ${ }^{50-12}$ Ultimately, the mechanical disruptions and inflammatory responses activate Type III and IV pain receptors, leading to the sensation of DOMS. ${ }^{13}$

Some strategies proposed to alleviate DOMS include pre and postexercise stretching, light exercise, ultrasound, topical analgesics, and pharmacological agents. None of these treatments, however, completely attenuate DOMS. ${ }^{14}{ }^{15}$ Muscle massage may be an alternative therapy, one that is popular and possibly effective.$^{16}$ If massage is rendered during the early stages of inflammation, the mechanical pressure applied with the massage might decrease neutrophil margination, thereby reducing inflammation and DOMS. ${ }^{16-18}$ Indeed, massage rendered $2 \mathrm{~h}$ after muscle injury decreased muscle soreness and increased the circulating neutrophil count, which suggests the treatment reduced neutrophil margination. ${ }^{17}$ Theoretically, decreased margination should have attenuated muscle damage induced by inflammation, a variable not measured in the aforementioned study. ${ }^{17}$ Muscle damage is frequently monitored in other studies by examining range of motion (ROM) and peak torque. ${ }^{19}$ Although these measures are indirect, the relationship among muscle damage, inflam- mation, and changes in muscle function is well documented. ${ }^{2}{ }^{19-21}$

Our primary purpose, therefore, was to track how massage affects circulating neutrophil levels, peak torque, ROM, and muscle soreness during DOMS. Data from those studies in which the effects of massage on muscle function and DOMS were examined are difficult to reconcile because treatment length, technique, and application time varied. ${ }^{1522-25}$ To circumvent some of these limitations, we used classical Swedish massage techniques shown to be effective in reducing soreness, ${ }^{172}$ and measured the muscle function tests at the same postexercise times as in previous research. ${ }^{20}{ }^{21}$ Additionally, to further extend the work of Smith et al, ${ }^{17}$ we used a more sophisticated measurement of soreness, the Differential Descriptor Scale (DDS). The DDS provides a more complete assessment of pain than simple scales, such as visual analog, numerical, or verbal, because it measures both the sensory and emotional aspects of pain. ${ }^{26}$ Simple scales, in contrast, treat pain solely as a one dimensional sensation, varying in intensity only. To combat another shortcoming of simple scales, the DDS descriptors are randomly arranged for each session and located above a line with 21 points. The descriptors on simple scales are not randomly placed, which makes them vulnerable to bias, as subjects tend to repeatedly use the same category or part of a line. ${ }^{26}$

Researchers also report that massage enhances perception of recovery after boxing performances, ${ }^{27}$ and improves mood, as measured by a Profile of Mood States (POMS). ${ }^{28}$ To date, researchers have not examined the possible relationship among massage, mood perceptions, and DOMS. Hence, our secondary purpose was to make a broader assessment of the psychological effects of massage on DOMS by using both the POMS and DDS analyses.

\section{METHODS}

\section{Subjects}

Eighteen male and female volunteers, with a mean (SD) for age and weight of 20.4 (1.0) years and 72.6 (14.1) kg respectively. None of the subjects were pregnant, participating in a competitive sport, recovering from a knee injury, taking antiinflammatory medication, or weight training the hamstrings 
in the four months preceding the study. All subjects signed an informed consent document and the Ithaca College Review Board for Human Subjects Research approved this project.

\section{Baseline tests}

Subjects reported to the lab one to two days prior to the experimental treatment for familiarisation and baseline measurements. Upon arrival they completed the POMS questionnaire so that baseline values for the following mood states could be established: Tension-Anxiety, Depression-Dejection, Anger-Hostility, Vigour-Activity, Fatigue-Inertia, and Confusion-Bewilderment. ${ }^{29}$ After the POMS, hamstring ROM was measured with a standard uni-level inclinometer during a straight leg raise. An average over three trials was recorded as the baseline ROM. ${ }^{30}$

After the ROM determination, subjects were familiarised with the isokinetic device (Cybex Norm, Version 2.01, Henly Healthcare, USA) that was used to measure peak torque and induce muscle soreness. Immediately after this familiarisation period, the subjects completed eight submaximal and two maximal eccentric contractions with the right hamstrings $\left(0-70^{\circ}\right.$ of extension) at a slow velocity $\left(30^{\circ} / \mathrm{s}\right)$ as a warm up for the baseline peak torque measurements. Subjects resisted the eccentric movement of the lever arm and observed visual feedback of torque to encourage maximal effort. After 2 min of rest, they completed five maximal eccentric contractions with the right hamstrings. The highest value obtained during these lifts was recorded as peak torque and served as the baseline value for the study.

After the peak torque determination, subjects completed the DDS, which contains two sets of 12 descriptor items that measure both the intensity and unpleasantness of soreness. ${ }^{26}$ As previously stated, the DDS descriptors are randomly arranged for each session and located above a line with 21 points for the subjects to mark. Scores for the respective descriptor sets were summed and averaged to obtain mean scores for intensity and unpleasantness of muscle soreness, which served as baseline values for this study.

After completing the DDS, a $5 \mathrm{ml}$ blood sample was taken by venipuncture from each subject. Differential slides were created, dried, and stained for 7 min with a Hematek 100 (Miles and Company, USA). A trained technician counted 100 cells and recorded the percentage of neutrophils. This count was the baseline measure for this study. Subjects were randomly assigned to one of two groups: massage intervention $(n=9)$ or control $(n=9)$ after the first lab visit.

\section{Experimental treatment}

The experimental treatment for each subject began at 8:00 am on the scheduled day and started with the previously described isokinetic warm up protocol. Afterwards, six sets of 10 maximal eccentric contractions with the right hamstring were completed to induce muscle damage; there was 1 min of rest between sets. Next, each subject completed five more maximal eccentric contractions. Peak torque was recorded from these additional lifts and labelled as $0 \mathrm{~h}$ postexercise.
The subjects returned at $2 \mathrm{~h}$ postexercise and peak torque was again measured as previously described. Next, the subjects received $20 \mathrm{~min}$ of massage or control treatment depending on their group assignment. The muscle massage consisted of classical Swedish techniques, which are preferred by most physiotherapists, ${ }^{31}$ and included: five minutes of effleurage (stroking), one minute of tapotement (percussion), 12 minutes of petrissage (kneading), and two additional minutes of effleurage. ${ }^{31} 32$ A senior physical therapy student, whose time on each stroke was standardised by an audiotape, performed the massage. The control treatment consisted of a placebo lotion that was applied to the control subjects' legs by the masseuse, who then instructed these subjects to rest for 20 min while listening to the same audiotape heard by the massage group. Subjects in both groups were told that their treatment might reduce inflammation. After the treatment, subjects completed a POMS questionnaire. At 6 and 24 h postexercise, subjects returned to the lab so that mood state, ROM, peak torque, soreness, and neutrophil levels could be measured. Final data collection without neutrophil assessment occurred at $48 \mathrm{~h}$ postexercise.

\section{Statistical analysis}

A two factor ANOVA (treatment $\times$ time) with repeated measures on the second factor was used for each dependent variable: peak torque, ROM, DDS intensity and unpleasantness of soreness, neutrophil levels, and POMS score. A Tukey posthoc test was used when a significant time effect was observed, whereas a Bonferroni posthoc test was used when a significant interaction was observed. Statistical significance was set at $\mathrm{p}<0.05$ for all tests.

\section{RESULTS}

Table 1 shows the values for hamstring peak torque and ROM. A main time effect for torque $(\mathrm{F}(5,80)=24.63, \mathrm{p}<0.000)$ and $\operatorname{ROM}(\mathrm{F}(3,48)=20.28, \mathrm{p}<0.000)$, but no treatment effect or interaction. Tukey posthoc analyses showed that both groups had a significant decrease in strength at each postexercise time, whereas ROM decreased significantly in both groups at 24 and $48 \mathrm{~h}$ postexercise. Since there was a significant baseline difference in the neutrophil data $(p<0.05)$, a two factor ANCOVA (treatment $\times$ time) was used to analyse them. The analysis indicated that there were no significant differences across time or between groups for this variable. The adjusted means (SD) for neutrophils per 100 leukocytes in the massage group at $6 \mathrm{~h}$ and $24 \mathrm{~h}$ were 61.5 (4.6) and 57.5 (3.3), respectively. The corresponding values for the control group were 54.1 (4.6) and 50.2 (3.3). The baseline prior to adjustment was 58.5 .

Figures 1 and 2 show the DDS intensity and unpleasantness data. There was a significant interaction $(\mathrm{F}(3,45)=3.02$, $\mathrm{p}<0.05)$ and main time effect $(\mathrm{F}(3,45)=45.57, \mathrm{p}<0.05)$ in the intensity data. Bonferroni posthoc analyses showed that both groups had significantly higher intensities of soreness at 6,24 , and $48 \mathrm{~h}$ postexercise; the analyses also indicated that

Table 1 Mean (SD) for changes in hamstring peak eccentric torque $(\mathrm{N} \cdot \mathrm{m})$ and range of motion (degrees)

\begin{tabular}{lllllll}
\hline $\begin{array}{l}\text { Variable/ } \\
\text { group }\end{array}$ & Baseline & $0 \mathrm{~h}$ & $2 \mathrm{~h}$ & $6 \mathrm{~h}$ & $24 \mathrm{~h}$ & $48 \mathrm{~h}$ \\
\hline $\begin{array}{l}\text { Peak torque } \\
\text { Massage }\end{array}$ & $144.7(49.2)$ & $120.3(38.2)^{*}$ & $110.5(35.6)^{*}$ & $115.5(36.7)^{*}$ & $110.2(36.7)^{*}$ & $115.8(38.2)^{*}$ \\
$\begin{array}{c}\text { Control } \\
\text { ROM }\end{array}$ & $154.4(42.4)$ & $134.3(43.1)^{*}$ & $123.6(41.2)^{*}$ & $128.7(44.8)^{*}$ & $119.2(46.3)^{*}$ & $120.9(44.8)^{*}$ \\
Massage & $88.9(17.8)$ & N/A & N/A & $84.8(12.8)$ & $79.3(10.7)^{*}$ & $79.7(14.0)^{*}$ \\
Control & $82.4(8.6)$ & N/A & N/A & $78.3(10.3)$ & $75.1(8.5)^{*}$ & $69.0(10.6)^{*}$ \\
\hline
\end{tabular}

*Indicates a significant difference from baseline $(p<0.00)$.

$\mathrm{N} / \mathrm{A}$ - Indicates that ROM was not measured during this time period. 


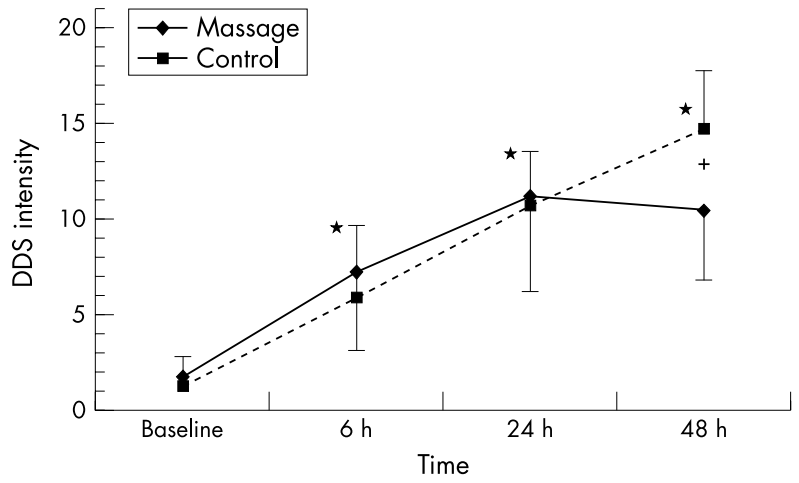

Figure 1 Differential Descriptor Scale intensity of soreness in massage and control subjects. Data are presented as means (SD), and higher scores represent greater intensities of soreness. Both groups indicated significantly higher ( ${ }^{*}=p \leqslant 0.000$ ) intensities of soreness at 6,24 , and $48 \mathrm{~h}$ postexercise compared to baseline. Also, the control group reported significantly greater

$(+=p \leqslant 0.000)$ intensity of soreness at $48 \mathrm{~h}$ postexercise compared to the massage group.

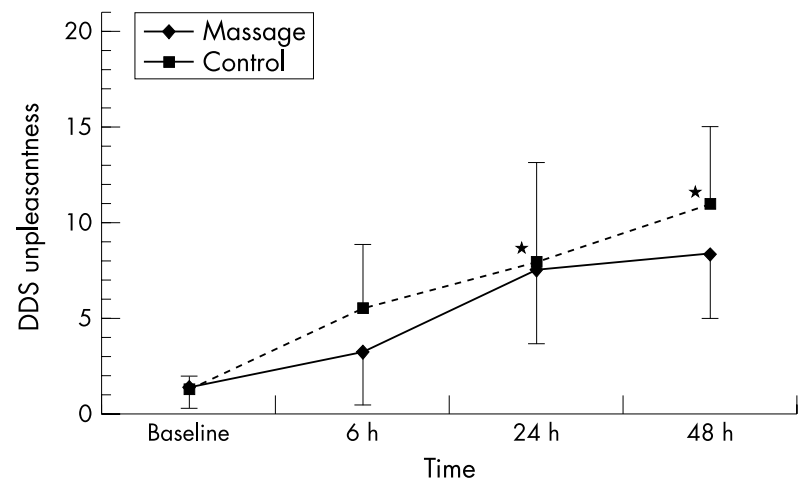

Figure 2 Differential Descriptor Scale unpleasantness of soreness in massage and control subjects. Data are presented as means (SD), and higher scores represent greater perceptions of unpleasantness. Both groups indicated significant increases $\left.{ }^{*}=p \leqslant 0.000\right)$ in unpleasantness at $24 \mathrm{~h}$ and $48 \mathrm{~h}$ postexercise compared to baseline.

the control group's soreness was more intense than the massage group's at $48 \mathrm{~h}$ postexercise (figure l). The only significant finding in the unpleasantness data was for time $(\mathrm{F}$ $(3,42)=34 \cdot 12, \mathrm{p}<0.05)$. A Tukey posthoc analysis indicated that both groups had higher feelings of unpleasantness at 24 and $48 \mathrm{~h}$ postexercise (figure 2). Figure 3 shows the POMS data. There was a main time effect $(\mathrm{F}(4,56)=3.144$, $\mathrm{p}<0.05)$, but no treatment effect or interaction. A Tukey posthoc test revealed that mood disturbance in both groups was significantly greater at $24 \mathrm{~h}$ postexercise than at baseline.

\section{DISCUSSION}

Our primary purpose was to assess the physiological effects of massage on DOMS. We found that massage rendered $2 \mathrm{~h}$ after strenuous eccentric exercise did not alter the circulating neutrophil count, peak torque, or ROM. Our finding that massage did not alter the neutrophil count was robust, as our power for this analysis was equal to 1.0. These data suggest that massage did not affect neutrophil margination, a finding that contrasts with data from Smith et al. ${ }^{17}$ One possible explanation for this discrepancy in the data is the quantity of muscle mass injured, as we studied a larger muscle mass than the previous study. ${ }^{17}$ Induced injury to a larger muscle mass could initiate a greater inflammatory response, possible making it more difficult to discern a treatment effect on neutrophil margination. The duration of the work bout used to induce DOMS also varied

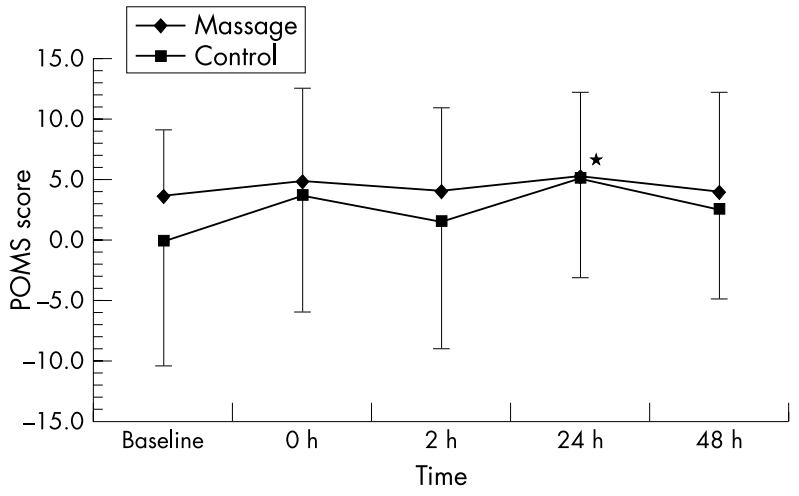

Figure 3 Profile of Mood States (POMS) scores in massage and control subjects. Data are presented as means (SD), and higher scores represent greater mood disturbances. Both groups indicated significantly higher $\left({ }^{*}=p<0.000\right)$ POMS scores at $24 \mathrm{~h}$ postexercise compared to baseline.

between the studies. Our subjects completed 60 maximal eccentric repetitions, compared to only 35 in the early work. The greater duration of exercise in our study may have induced more muscle damage, a hypothesis that could be supported by muscle function data. The earlier study, however, did not measure changes in muscle function, which prevents such a comparison.

Similar to Smith et al, ${ }^{17}$ we inferred neutrophil margination from differences in the circulating levels of neutrophils. Our inference is further limited because we estimated neutrophil levels by measuring their percentage in a sample of 100 leukocytes as opposed to determining the total and differential white blood cell counts. To definitively assess massage's effects on the neutrophil count after induced muscle injury, changes in neutrophil distribution and function, including migration, adherence, phagocytosis, and degranulation should be tracked. ${ }^{11} 33$ 34

Based on the current theory for DOMS, ${ }^{1-3} 2021$ the inability of massage to alter circulating neutrophil levels suggests that muscle damage induced by inflammation would not be attenuated. Indeed, we found that massage did not alter muscle function between the groups, which provides additional evidence that neutrophil margination was unaltered. Our ability to draw strong conclusions from the muscle function data is limited because of large variability, but these findings are consistent with peak torque measurements in other studies. ${ }^{20}{ }^{21}$ Power analyses revealed that given the variability in our data, we would have needed a sample size greater than 55 to achieve a power $=0.8$.

Our secondary purpose was to determine the psychological effects of massage on DOMS. In line with data from other studies, massage did not affect the unpleasantness of soreness, which bolsters the supposition that this dimension of the DDS scale does not figure prominently in DOMS. ${ }^{20}{ }^{21}$ As reflected by the POMS data, massage also did not significantly affect mood. This finding contrasts with data from a study in which massage improved mood following physical exertion. ${ }^{27}$

As suggested, the effects of massage may indeed by more psychological than physiological..$^{27}{ }^{32}{ }^{35}$ We found support for this hypothesis, as massage lowered soreness intensity at $48 \mathrm{~h}$ postexercise. Overall power for this analysis was not particularly robust $(0.52)$, but it did increase substantially to 0.75 at $48 \mathrm{~h}$. This level of power is consistent with the body of work completed on human subjects ${ }^{36}$ and indicates that the probability of making a type II error is less than $25 \%$. However, it is difficult to draw definitive conclusions because we only found one significant time point and the overall variability was high. Nevertheless, because the data are similar to those from Smith et al, ${ }^{17}$ there is a growing body of evidence showing massage lowers the intensity of soreness during DOMS. 


\section{Take home message}

Although massage rendered after muscle injury did not alter any physiological variables, it did lower the intensity of soreness after $48 \mathrm{~h}$.

Possible explanations for how massage decreases muscle soreness include improved sleep patterns, increased endorphin and serotonin levels, and decreased levels in stress hormones following treatment. ${ }^{32}{ }^{35}{ }^{37}$ Additionally, massage may activate pressure instead of pain receptors, thereby lowering soreness intensity. ${ }^{13}$

\section{CONCLUSION}

Massage administered after DOMS inducing exercise did not alter neutrophil levels or hamstring function. Massage, however, did lower the intensity of soreness. The mechanism for the difference in soreness intensity remains to be elucidated.

\section{ACKNOWLEDGEMENTS}

We would like to thank Joseph Murray for the massage treatments and the Ithaca College Physical Therapy Department. Also, we are greatly indebted to Janet Wigglesworth, whose statistical analyses and advice were instrumental to this project.

\section{REFERENCES}

1 Armstrong RB. Mechanisms of exercise-induced delayed onset muscular soreness: a brief review. Med Sci Sports Exerc 1984;16:529-38.

2 Maclntyre DL, Reid WD, McKenzie DC. Delayed onset muscle soreness: the inflammatory response to muscle injury and its clinical implications. Sports Med 1995;20:24-40.

3 Powers SK, Howley ET. Exercise physiology: theory and application to fitness and performance. 3rd ed. Baltimore, MD: Williams \& Waverly, 1996.

4 Smith LL. Acute inflammation: the underlying mechanisms in delayed onset muscle soreness? Med Sci Sports Exerc 1991;23:542-51.

5 Clarkson PM, Sayers SP. Etiology of exercise-induced muscle damage. Can J Appl Physiol 1999;24:234-48.

6 Child RB, Saxton JM, Donnelly AE. Comparison of eccentric knee extensors muscle actions at two muscle lengths on indices of damage and angle-specific force production in humans. J Sports Sci 1998;16:301-8.

7 Newman DJ, Jones DA, Ghosh G, et al. Muscle fatigue and pain after eccentric contractions at long and short muscle lengths. Clin $\mathrm{Sci}$ 1988;74:553-7.

8 Belcastro AN. Skeletal muscle calcium-activated neutral protease (calpain) with exercise. J Appl Physiol 1993;74:1381-6.

9 Belcastro AN, Shewchuk LD, Raj DA. Exercise-induced muscle injury: a calpain hypothesis. Mol Cell Biochem 1998;179:135-45.

10 Faulkner JA, Brooks SV, Opiteck JA. Injury to skeletal muscle fibers during contractions: conditions of occurrences and prevention. Phys Ther 1993;73:911-21.

11 Weiss SJ. Tissue destruction by neutrophils. N Engl J Med $1989 ; 320: 365-76$
12 Edwards SW. Biochemistry and physiology of the neutrophil. New York: Cambridge University Press, 1994.

13 O'Conner PJ, Cook DB. Exercise and pain: the neurobiology, measurement, and laboratory study of pain in relation to exercise in humans. Exerc Sport Sci Rev 1999;27:1 19-66.

14 Bougie JD. Management of delayed onset muscle soreness: a review of literature. Sports Chiropractic and Rehabilitation 1997;11:1-10.

15 Gulick DT, Kimura IF, Sitler M, et al. Various treatment techniques on signs and symptoms of delayed onset muscle soreness. Journal of Athletic Training 1996;31:145-52.

16 Ernst E. Does post-exercise massage treatment reduce delayed onset muscle soreness? A systematic review. Br J Sports Med 1998;32:212-14.

17 Smith LL, Keating MN, Holbert D, et al. The effects of athletic massage on delayed onset muscle soreness, creatine kinase, and neutrophil count: a preliminary report. Journal of Orthopedic and Sport Physical Therapy 1994;19:93-9.

18 Tiidus PM. Massage and ultrasound as therapeutic modalities in exercise-induced muscle damage. Can J Appl Physiol 1999;24:267-78.

19 Warren GL, Lowe DA, Armstrong RB. Measurement tools used in the study of eccentric contraction-induced injury. Sports Med 1999;27:43-59.

20 Maclntyre DL, Reid WD, Lyster DM, et al. Presence of WBC, decreased strength, and delayed soreness in muscle after eccentric exercise. J Appl Physiol 1996;80:1006-13

21 Maclntyre DL, Reid WD, Lyster DM, et al. Different effects of strenuous eccentric exercise on the accumulation of neutrophils in muscle in women and men. Eur J Appl Physiol 2000;81:47-53.

22 Cafarelli E, Sim J, Carolan B, et al. Vibratory massage and short-term recovery from muscular fatigue. Int J Sports Med 1990;1 1:474-8.

23 Rodenburg JB, Steenbeck P, Shiereck P, et al. Warm-up, stretching, and massage diminish the harmful effects of eccentric exercise. Int J Sports Med 1994;15:414-19.

24 Shoemaker JK, Tiidus PM, Mader R. Failure of manual massage to alter limb blood flow: measure by Doppler ultrasound. Medicine and Science in Sport and Exercise 1997;29:610-14.

25 Viitasalo JT, Niemela K, Kaappola R, et al. Warm underwater water-jet massage improves recovery from intense physical exercise. Eur J Appl Physiol 1995;71:431-8.

26 Gracely RH, Kwilosz DM. The Descriptor Differential Scale: applying psychophysical principles to clinical assessment. Pain 1988;35:279-88.

27 Hemmings B, Smith M, Graydon J, et al. Effects of massage on physiological restoration, perceived recovery, and repeated sports performance. Br J Sports Med 2000;34:109-15.

28 Weinberg AJ, Kolodny K. The relationship of massage and exercise to mood enhancement. The Sport Psychologist 1988:2:202-11.

29 Mcnair DM, Lorr ML, Droppleman LF. EITS manual for the Profile of Mood States. San Diego, CA, USA: Educational and Industrial Testing Service, 1981.

30 Gerhardt J. Documentation of joint movement. 3rd ed. Portland, OR, USA: Isomed, 1992

31 Callaghan MJ. The role of massage in the management of the athlete: a review. Br J Sports Med 1993;27:28-33.

32 Cafarelli $E$, Flint $F$. The role of massage in preparation for and recovery from exercise: an overview. Sports Med 1992;14:1-9.

33 Pyne DP. Regulation of neutrophil function during exercise. Sports Med 1994:17:245-58

34 Croisier JL, Camus G, Deby-Dupont G, et al. Myocellular enzyme leakage, polymorphonuclear neutrophil activation and delayed onset muscle soreness induced by isokinetic eccentric exercise. Arch Physiol Biochem 1996;104:322-29.

35 Field T. Massage therapy effects. Am Psychol 1998;53:1270-81.

36 Sterne JAC, Smith GD. Sifting the evidence-what's wrong with significance tests? Phys Ther 2001;81:1464-9.

37 Goldfarb AH, Jamurtas AZ. Beta endorphin response to exercise: an update. Sports Med 1997;24:8-16. 